\title{
ESTUDO COMPARATIVO DE TRÊS MECANISMOS DE REDUÇÃO DE EVAPORAÇÃO DE RESERVATÓRIOS
}

\author{
Fernanda Helfer ${ }^{1}$, José Antônio Louzada ${ }^{2}$, Hong Zhang ${ }^{3}$, Charles Lemckert ${ }^{4}$ e \\ Nilza Maria dos Reis Castro ${ }^{5}$
}

\begin{abstract}
Resumo: $O$ uso de tecnologias para redução da evaporação de reservatórios pode ajudar regiões áridas $e$ semiáridas a superar a escassez hídrica. Alguns mecanismos, como filmes monomoleculares e estruturas flutuantes, podem apresentar efeitos negativos nos ambientes aquáticos e na qualidade da água. Este trabalho investigou a eficácia de três mecanismos de redução de evaporação que possuem alto desempenho ambiental: coberturas suspensas, destratificadores e quebra-ventos. A análise foi conduzida com o modelo DYRESM em um reservatório localizado no sudeste do estado de Queensland, Austrália. O modelo foi primeiramente calibrado e validado para o reservatório em estudo. Em seguida, as condições de contorno foram alteradas para refletir os efeitos dos mecanismos de redução de evaporação no comportamento térmico do reservatório. O método mais eficaz foi o uso de coberturas suspensas, que reduziu em $88 \%$ a evaporação. O uso de quebra-ventos reduziu em $27 \%$ a evaporação, e o uso de destratificadores não teve efeito significativo na redução da evaporação. Para cada um dos mecanismos estudados, uma análise compreensiva dos seus efeitos na temperatura da água e nas taxas de evaporação foi efetuada, inferindo sobre os potenciais resultados do uso destes mecanismos em reservatórios de outras condições climáticas.
\end{abstract}

Palavras-chave: Evaporação. DYRESM. Coberturas suspensas. Destratificadores. Quebra-ventos.

\section{Introdução}

Diversas regiões do globo enfrentam uma pressão crescente sobre os recursos hídricos devido ao forte crescimento populacional, o desenvolvimento econômico, $\mathrm{e}$ às consequências impostas ao meio ambiente pelas alterações climáticas globais. A agricultura é, sem dúvida, um dos setores que mais sofre com esta pressão, especialmente em regiões áridas $e$ semiáridas, onde o problema de escassez hídrica é mais grave (PAZ; TEODORO; MENDONÇA, 2000; ROSEGRANT; RINGLER; ZHU, 2009; MAESTRE-VALERO et al., 2013). A preocupação com a disponibilidade de água para agricultura tem levado muitos países áridos e semiáridos a desenvolverem mecanismos para redução de perdas e para aumento de disponibilidade de água para irrigação (PEREIRA et al., 2009). Tecnologias para reduzir as taxas de evaporação de reservatórios agrícolas são exemplos de mecanismos que podem efetivamente ajudar estes países e regiões a superar a escassez de água, e consequentemente aumentar o rendimento das culturas agrícolas (MARTíNEZ ALVAREZ et al., 2008; GALLEGO-ELVIRA et al., 2013; SADEGHI et al., 2015).

Na Austrália, por exemplo, onde 70\% da área é ocupada por terras áridas ou semiáridas, a evaporação de reservatórios agrícolas é considerada o pior fator de contribuição para a perda de água em zonas rurais. $O$ volume de água armazenado nos reservatórios agrícolas australianos responde por 9\% (cerca de $7.000 \mathrm{hm}^{3}$ ) do total de água armazenado no país. No entanto, estima-se que $40 \%$ desse volume é evaporado a cada ano (CRAIG et al., 2005). Nas regiões áridas e semiáridas da Austrália, a evaporação de reservatórios excede 2000 $\mathrm{mm}$ por ano (DEPARTMENT OF NATURAL RESOURCES AND MINES, 2002); consequentemente, a disponibilidade de água para os cultivos diminui, afetando a produtividade e a renda agrícola. Além disso, as mudanças climáticas tem sido uma ameaça expressiva para a disponibilidade de

\footnotetext{
'E-mail: f.helfer@griffith.edu.au

2E-mail: louzada@iph.ufrgs.br

Instituto de Pesquisas Hidráulicas, UFRGS. Av. Bento Gonçalves 9500. Caixa postal 15029. CEP: 91501-970. Porto Alegre - RS

${ }^{3}$ E-mail: hong.zhang@griffith.edu.au

${ }^{4}$ E-mail: charles.lemckert@canberra.edu.au

5*E-mail: nilza@iph.ufrgs.br
} 


\section{REA - Revista de estudos ambientais (Online) v.20, n. 2, p.62-76, jul./dez. 2018}

água nos reservatórios australianos. De acordo com o Csiro e Bom (2007) e Johnson e Sharma (2010), há muita evidência científica indicando aumentos expressivos nas temperaturas e nas taxas de evaporação na Austrália. Em 2070, estima-se que a evaporação sofrerá um aumento de $6 \%$ no Sul e no Oeste da Austrália, e de $10 \%$ no Norte e no Leste em comparação com a evaporação média de 1990-2010. No estado de Queensland, onde este estudo foi realizado, uma investigação recente mostrou que a evaporação anual será aproximadamente $8 \%$ maior do que a evaporação média anual atual em torno do ano de 2040 , e $15 \%$ maior em todo o ano de 2080 (HELFER; LEMCKERT; ZHANG, 2012a).

Embora o Brasil seja considerado um país rico em disponibilidades hídricas, observa-se uma enorme desigualdade regional na distribuição dos recursos hídricos (PAZ; TEODORO; MENDONÇA, 2000; FONTES; OLIVEIRA; MEDEIROS, 2003), com muitas regiões assemelhando-se às regiões áridas e semiáridas da Austrália, como o Nordeste brasileiro, que apresenta baixas taxas de precipitação e elevadas taxas de evaporação que frequentemente excedem $2000 \mathrm{~mm}$ por ano (REBOUÇAS, 1997; SILANS, 2003; LEÃO et al., 2013). Segundo Fontes, Oliveira e Medeiros (2003), a evaporação no Nordeste brasileiro varia de $1.000 \mathrm{~mm}$ por ano no litoral da Bahia a Recife, atingindo $2.000 \mathrm{~mm}$ por ano no interior, sendo que em Petrolina (Pernambuco), a evaporação chega a 3.000 $\mathrm{mm}$ por ano. De acordo com Suassuna (2002), o semiárido brasileiro engloba cerca de 70 mil açudes de pequeno porte (com volumes entre 10.000 e $200.000 \mathrm{~m}^{3}$ ) que representam $80 \%$ dos corpos d'água nos estados do Nordeste, e estima-se que cerca de $40 \%$ das águas acumuladas nestes reservatórios é perdida por evaporação. Aliado a isso, estudos apontam para uma redução significativa na disponibilidade hídrica desta região devido as mudanças climáticas globais (MELO BRANCO; SUASSUNA; VAINSENCHER, 2005). Este quadro no Nordeste brasileiro assemelha-se muito ao quadro Australiano, indicando que medidas tomadas por este país para aumentar a disponibilidade hídrica em zonas rurais poderiam ser transferidas com sucesso para o combate da escassez hídrica no semiárido brasileiro.

O estudo e o desenvolvimento de mecanismos de redução de evaporação de reservatórios são uma área prioritária na Austrália. Autoridades australianas estão constantemente buscando métodos de combater a evaporação de reservatórios (WATTS, 2005; ABC Rural, 2015; DEPARTMENT OF PRIMARY INDUSTRIES, 2015). Neste país, a redução de evaporação é vista como uma das poucas áreas em que poupanças significativas de água podem ser atingidas. Os métodos mais difundidos são 0 uso de filmes monomoleculares (BARNES, 1993; PRIME et al., 2012; GALLEGOELVIRA et al., 2013), corpos artificiais flutuantes à superfície (CRAIG, 2005; CRAIG; SCHMIDT; SCOBIE, 2006; YAO et al., 2010), coberturas suspensas (WATTS, 2005; ALVAREZ et al., 2006; CRAIG; SCHMIDT; SCOBIE, 2006; SCHMIDT, 2007), e quebra-ventos (HIPSEY, 2002; WATTS, 2005; HELFER; ZHANG; LEMCKERT, 2009). Outros métodos bastante estudados, mas não muito difundidos, são os usos de destratificadores para mistura vertical (HELFER; LEMCKERT; ZHANG, 2011b; HELFER; LEMCKERT; ZHANG, 2012b) e a redução da relação entre área superficial e volume de reservatórios (MCJANNET; COOK; BURN, 2008).

Coberturas suspensas são estruturas sombreadoras suspensas acima da superfície da água e suportadas externamente por cabos e postes. A cobertura reduz a evaporação principalmente através do bloqueio da radiação solar incidente sobre a superfície da água, reduzindo assim a temperatura da superfície (ALVAREZ et al., 2006; FINN e BARNES, 2007). As coberturas suspensas também ajudam a reduzir a ação do vento sobre o vapor de água na superfície dos lagos, mantendo a umidade acima da superfície e diminuindo assim o gradiente de pressão de vapor sobre a água e, consequentemente, diminuindo a taxa de evaporação. Esses fatores combinados ajudam a reduzir em até $75 \%$ as taxas de evaporação sem afetar a qualidade da água, a entrada da precipitação e a vida aquática (WATTS, 2005; ALVAREZ et al., 2006; CRAIG; SCHMIDT; SCOBIE, 2006; SCHMIDT, 2007). Estas estruturas são uma opção prática para pequenos açudes onde as distâncias de apoio usadas na ancoragem são relativamente curtas (WATTS, 2005).

Destratificadores de reservatórios são dispositivos mecânicos criados para reduzir a estratificação térmica e aumentar a transferência vertical de oxigénio dissolvido na coluna de água. Consistem de um difusor 


\section{REA - Revista de estudos ambientais (Online) v.20, n. 2, p.62-76, jul./dez. 2018}

instalado no fundo do reservatório com um certo número de portas, por onde o ar é injetado na água. $\mathrm{O}$ ar injetado cria bolhas que arrastam consigo a água do fundo do reservatório, que se mistura com a água mais quente da superfície. Em reservatórios que sofrem estratificação térmica, onde as temperaturas do fundo do reservatório são significativamente mais frias que as da superfície, este processo pode posteriormente reduzir as taxas de evaporação devido à redução da temperatura da água superficial (HELFER; LEMCKERT; ZHANG, 2011a; HELFER; LEMCKERT, C.; ZHANG, 2011b). Estes sistemas somente são eficazes em reduzir evaporação em reservatórios que apresentam marcante estratificação térmica, com diferenças de temperatura entre superfície e fundo bastante acentuadas (HELFER; LEMCKERT; ZHANG, 2011a; HELFER; LEMCKERT, C.; ZHANG, 2011b).

Os quebra-ventos são estruturas (barreiras físicas) que têm potencial em reduzir a evaporação devido a seu efeito na velocidade do vento e umidade do ar, a jusante da barreira. O nível de alteração da velocidade do vento causado pela presença de barreiras é função da altura, densidade e orientação da barreira em relação ao vento predominante. Estas barreiras reduzem a velocidade do vento até sobre determinada distância à jusante do quebra-vento, a qual depende da altura da barreira (WANG; TAKLE, 1997; HIPSEY, 2002; HELFER; ZHANG; LEMCKERT, 2009). Com esta redução na velocidade do vento, a taxa de remoção de umidade sobre a superfície da água também diminui, diminuindo 0 gradiente de umidade na superfície e, consequentemente, as taxas de evaporação (HELFER; ZHANG; LEMCKERT, 2009). A eficiência de um quebra-vento na redução da velocidade do vento depende principalmente da sua permeabilidade, da altura e da orientação em relação ao vento predominante (WANG; TAKLE, 1997; HIPSEY, 2002; SUDMEYER; SCOTT, 2002; SUDMEYER; BICKNELL; COLES, 2007).

O DYRESM é um modelo computacional usado para estimar a distribuição vertical da temperatura da água e o balanço hídrico de lagos e reservatórios em intervalos de tempo diários ou subdiários. Este modelo tem sido usado amplamente em uma grande variedade de lagos e reservatórios com diferentes morfologias e condições climáticas (PERROUD et al., 2009; HELFER;
LEMCKERT; ZHANG, 2012a; MCGLOIN et al., 2014; WEINBERGER; VETTER, 2014). Embora o modelo possa ser usado para estimar os fluxos de calor latente por evaporação (HELFER; LEMCKERT; ZHANG, 2012a; MCGLOIN et al., 2014), a grande parte de suas aplicações está relacionada à ecologia e qualidade de água de reservatórios.

As variáveis de entrada principais do modelo DYRESM são a morfometria do lago (representada pela relação área versus profundidade), a meteorologia (radiação solar, vento, temperatura do ar, umidade do ar e precipitação) e os volumes de entrada e saída (afluentes e retiradas). As variáveis de saída são a temperatura da água, salinidade e densidade em diversas profundidades. Como todo modelo unidimensional de reservatórios, o modelo DYRESM considera que as variações verticais na densidade, temperatura e salinidade são mais importantes que as variações horizontais, uma vez que estas são relaxadas rapidamente devido à força de restauração da estratificação ser superior do que a força imposta pelo vento e pela entrada de afluentes.

O modelo usa uma representação do reservatório em camadas horizontais, onde não há variação das propriedades (temperatura, densidade e salinidade) no sentido lateral e longitudinal, apenas na vertical. Os vários parâmetros utilizados pelo modelo são baseados em processos físicos conhecidos e requerem pouco ou nenhum ajuste pelo usuário.

O presente estudo teve por objetivo investigar a eficácia de coberturas suspensas, destratificadores para mistura vertical e de quebra-ventos para reduzir a evaporação de pequenos reservatórios. O modelo unidimensional DYRESM (IMBERGER; PATTERSON, 1981; IMERITO, 2009a; b) foi aplicado à um reservatório de irrigação localizado no Sudeste de Queensland, Austrália, para simular a alteração da temperatura e da taxa de evaporação sob a utilização de tais mecanismos de redução de evaporação.

\section{Metodologia}

\section{1 Área de estudo}

A área deste estudo foi um reservatório de irrigação localizado no Sudeste de Queensland, Austrália, chamado 


\section{REA - Revista de estudos ambientais (Online) v.20, n. 2, p.62-76, jul./dez. 2018}

reservatório Logan (coordenadas 2734'26" S, $152^{\circ} 20^{\prime} 27^{\prime \prime} \mathrm{E}$, altitude $88 \mathrm{~m}$, Figura 1). O reservatório Logan tem uma capacidade de armazenamento de $0,7 \mathrm{hm}^{3}$, uma área superficial de 17 hectares quando cheio, e uma profundidade máxima de $6,5 \mathrm{~m}$, com profundidade média de $4,0 \mathrm{~m}$. O reservatório tem forma retangular, com dimensões de cerca de 480 metros por 350 metros.

Figura 1 - Vista aérea do reservatório de irrigação utilizado neste estudo e sua localização geral no estado de Queensland, Austrália. Coordenadas do centro de reservatório: 27034'26" S, 152020'27" E. Superfície: 17 hectares $\left(170.000 \mathrm{~m}^{2}\right)$

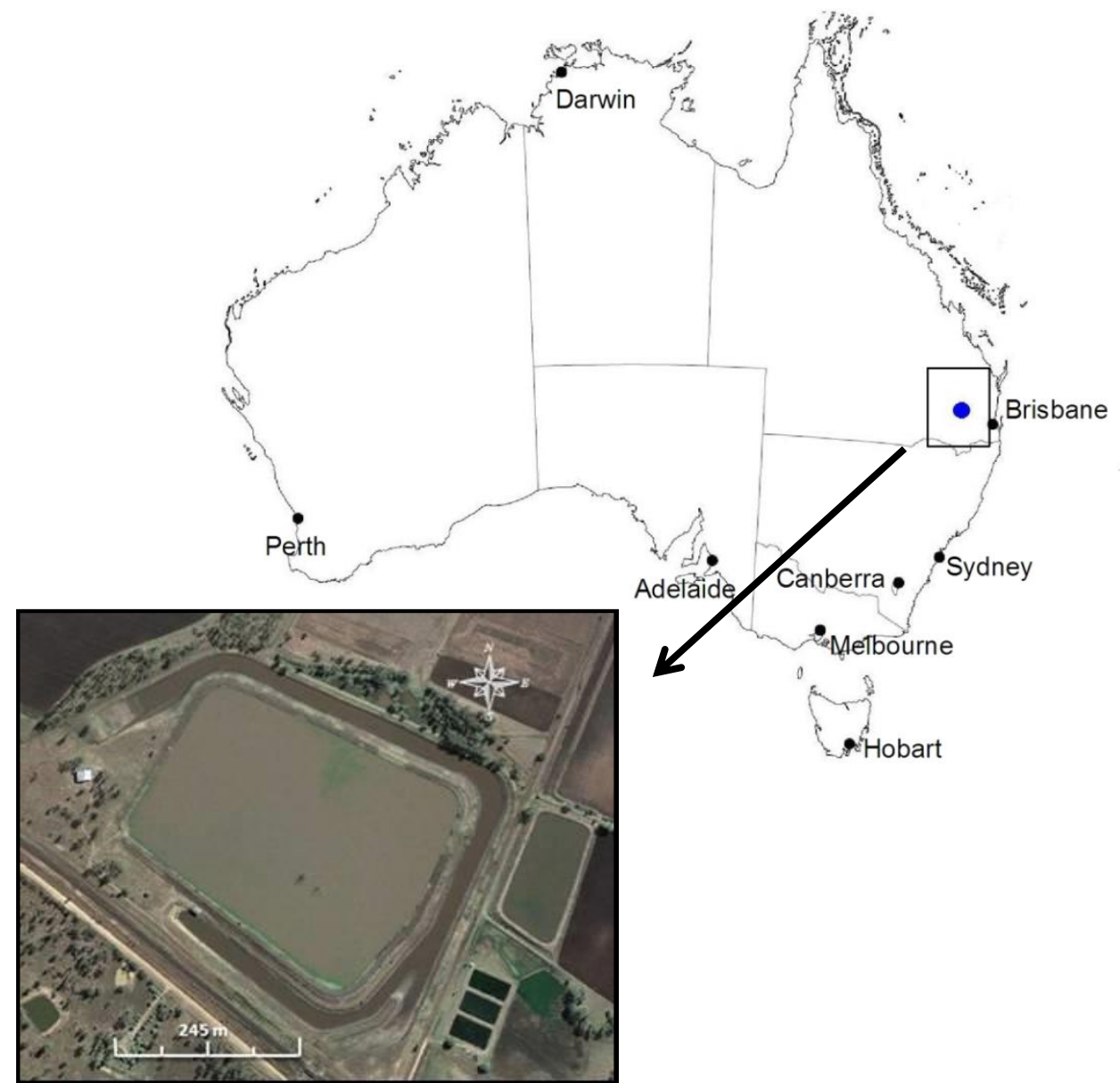

Fonte: Adaptado de Google Earth (2018)

O reservatório Logan foi objeto de estudo do projeto Evaporation Loss conduzido pela Urban Water Security Research Alliance (UWSRA) de 2007 a 2012 (URBAN WATER SECURITY RESEARCH ALLIANCE, 2012). De setembro de 2009 a abril de 2011 (18 meses), a UWSRA monitorou as variáveis climáticas do local e as perdas por evaporação do reservatório a fim de validar uma série de modelos de evaporação e de estudar uma série de mecanismos de redução de evaporação. Como parte desta investigação, equipamentos de monitoramento e medições, incluindo medidores de vazões (para monitorar e medir as entradas e saídas de água do reservatório), sensores de pressão (para monitorar e medir o nível de água no reservatório), sensores de temperatura (para monitorar e medir a temperatura da água em diversas profundidades e em diversos pontos do reservatório), estações meteorológicas (para monitorar e medir os parâmetros atmosféricos), e um equipamento de medição de fluxo por covariância de vórtices turbulentos (para medição direta de evaporação), foram instalados no reservatório. Uma descrição completa dos equipamentos utilizados e das variáveis monitoradas no Reservatório Logan é fornecida em Mcjannet et al. (2013), Mcgloin et al. (2014), Mcgloin, Mcgowana e Mcjannet (2015). Os dados necessários para alimentar 


\section{REA - Revista de estudos ambientais (Online) v.20, n. 2, p.62-76, jul./dez. 2018}

o modelo usado neste estudo foram todos fornecidos pela UWSRA.

O clima do Sudeste de Queensland, onde o reservatório está localizado, é classificado como Cfa ou subtropical, com verões quentes e úmidos e invernos frios e secos. No entanto, o reservatório Logan experimenta uma variedade de condições meteorológicas em diversas escalas espaciais e temporais (MCGLOIN; MCGOWANA; MCJANNET, 2015). Durante o período de monitoramento da UWSRA no reservatório Logan (outubro de 2009 a abril de 2011), a umidade relativa média foi de $75 \%$, com muito pouca variação sazonal. As temperaturas médias do ar variaram de 13,9 ${ }^{\circ} \mathrm{C}$ durante o inverno (junho-agosto) a 24,6 ${ }^{\circ} \mathrm{C}$ durante o verão (dezembro-fevereiro), com máxima temperatura média diária registrada em torno de $40^{\circ} \mathrm{C}$ em janeiro de 2010, e mínima temperatura registrada em torno de $1,5{ }^{\circ} \mathrm{C}$, em julho de 2010. A radiação solar média variou de $147 \mathrm{~W} \mathrm{~m} \mathrm{~m}^{-2}$ durante o inverno a $244 \mathrm{~W} \mathrm{~m}^{-2}$ durante $\mathrm{o}$ verão. A velocidade do vento não mostrou muita variação sazonal. A velocidade média foi de 2,7 m.s. ${ }^{-1}$. Por mais de $80 \%$ do tempo, a velocidade do vento foi inferior a $4 \mathrm{~m}^{-\mathrm{s}^{-1}}$. A direção predominante do vento foi do sudeste (30\% do tempo), seguida de leste (23\% do tempo), oeste (17\%), sul (15\%) e sudoeste $(10 \%)$. Ventos vindo do Norte foram observados em ocasiões raras (cerca de $5 \%$ do tempo). Durante os 18 meses de monitoramento, o total precipitado foi de $1.761 \mathrm{~mm}$, sendo que $62 \%$ deste total ocorreu nos meses de verão.

\subsection{0 modelo DYRESM}

O modelo DYRESM, tem como principais mecanismos de aquecimento, mistura e de estratificação do reservatório as trocas de calor, massa e momento. Estas trocas envolvem: i) aquecimento do lago por radiação de onda curta (dividida em duas porções - uma que fica retida na superfície, e outra que penetra no lago); ii) aquecimento por radiação de onda longa; iii) fluxo de quantidade de movimento (tensão de atrito); iv) fluxo de calor sensível; e v) fluxo de calor latente, ou de vapor de água (evaporação). Para os fluxos de quantidade de movimento, calor sensível e calor latente entre a superfície da água e a atmosfera, o modelo utiliza formulação aerodinâmica de transferência de massa (FISHER et al., 1979). Para o fluxo de vapor (calor latente), utilizado no cálculo da evaporação, a fórmula aerodinâmica utilizada pelo modelo é:

$$
\mathrm{Q}_{\mathrm{E}}=\mathrm{p}_{\mathrm{a}} \lambda \mathrm{C}_{\mathrm{E}} \mathrm{U}\left(\mathrm{q}_{\mathrm{a}}-\mathrm{q}_{\mathrm{s}}\right)
$$

onde $Q_{E}$ é o fluxo de calor latente devido à evaporação; $\rho_{a}$ é a densidade do ar $(=1,2 \mathrm{~kg}$ $\left.\mathrm{m}^{-3}\right), \lambda$ é o calor latente de vaporização (= $\left.2,453.10^{6} \mathrm{~J} \mathrm{~kg}^{-1}\right)$; CE é o coeficiente de transferência de calor $\left(=1,3 \cdot 10^{-3}\right)$, U é a velocidade do vento à altura de referência de $10 \mathrm{~m}$; qa é a umidade específica no ar; e qs é a humidade de saturação do ar, que é função da temperatura da água da superfície do reservatório. A perda de massa devido ao fluxo por evaporação é calculada como:

$$
\Delta \mathrm{M}=-\frac{\mathrm{Q}_{\mathrm{E}} \mathrm{A}}{\lambda}
$$

onde $\Delta M$ representa a variação da massa na camada superficial devido à evaporação e $A$, a área da superfície.

Para os demais aspectos do modelo DYRESM, o leitor pode consultar os manuais científico e de usuário do modelo (IMERITO, 2009a; b).

\subsection{Calibração e verificação do modelo DYRESM}

O modelo DYRESM foi calibrado com base na sua capacidade de reproduzir os valores observados de temperatura da água do reservatório Logan. O desempenho do modelo foi testado através da realização de análises de regressão entre as temperaturas diárias medidas e as temperaturas diárias estimadas pelo modelo. A calibração foi feita manualmente, utilizando-se o método de minimização de erros (PERROUD et al., 2009; WEINBERGER; VETTER, 2012). O período escolhido para a calibração foi de 29/09/2009 a 06/01/2010 (100 dias), cobrindo uma parte significativa da primavera e também do verão. Um processo de validação foi então realizado para o período de 07/01/2010 a 11/04/2010 (95 dias). O coeficiente de extinção de luz foi o único parâmetro utilizado na calibração do modelo. Este coeficiente influencia a absorção de calor na coluna de água (KLING, 1988), e tem uma sensibilidade significativa nas saídas do modelo. Um valor anual médio foi assumido nas simulações. Os valores testados variaram de 0,3 a 2,0 $\mathrm{m}^{-1}$ (KIRK, 2003; TANENTZAP; HAMILTON; YAN, 2007), com 


\section{REA - Revista de estudos ambientais (Online) v.20, n. 2, p.62-76, jul./dez. 2018}

$1,3 \mathrm{~m}^{-1}$ produzindo o melhor ajuste entre as temperaturas simuladas pelo modelo e as temperaturas medidas a campo. Este valor é realista, uma vez que o reservatório Logan é relativamente raso e sua turbidez é alta (OLIVER et al., 2000).

O coeficiente de determinação $\left(R^{2}\right)$ para o ajuste, considerando 100 dias de dados medidos, foi de 0,91 e a raiz do erro quadrático médio (RMSE) foi de $0,7^{\circ} \mathrm{C}$, com um erro médio de $-0,39{ }^{\circ} \mathrm{C}$. Estes índices demonstram que o modelo proporcionou uma representação satisfatória das temperaturas reais do reservatório Logan utilizando-se aquele valor de coeficiente de extinção de luz.
Sendo a temperatura da superfície da água o parâmetro principal no cálculo da evaporação, esta variável também foi considerada na avaliação do desempenho do modelo DYRESM. A Figura 2 mostra um coeficiente de determinação $\left(R^{2}\right)$ igual a 0,96 entre as temperaturas da superfície estimadas pelo modelo e as temperaturas medidas nos 100 dias considerados para calibração. A equação de regressão apresentou inclinação ligeiramente menor que $1,0(0,98)$, indicando que o modelo, em geral, tende a subestimar ligeiramente as temperaturas da superfície. No entanto, a raiz do erro quadrático médio foi de apenas $0,58{ }^{\circ} \mathrm{C}$, e o erro médio foi praticamente igual a zero $\left(-0,02{ }^{\circ} \mathrm{C}\right)$.

Figura 2 - Correlação entre temperaturas superficiais medidas (eixo $\mathrm{x}$ ) e temperaturas superficiais estimadas pelo modelo DYRESM (eixo y) no reservatório Logan. A linha sólida representa a equação de regressão, e a linha pontilhada, a reta $y=x$

Fonte: Autores (2018)

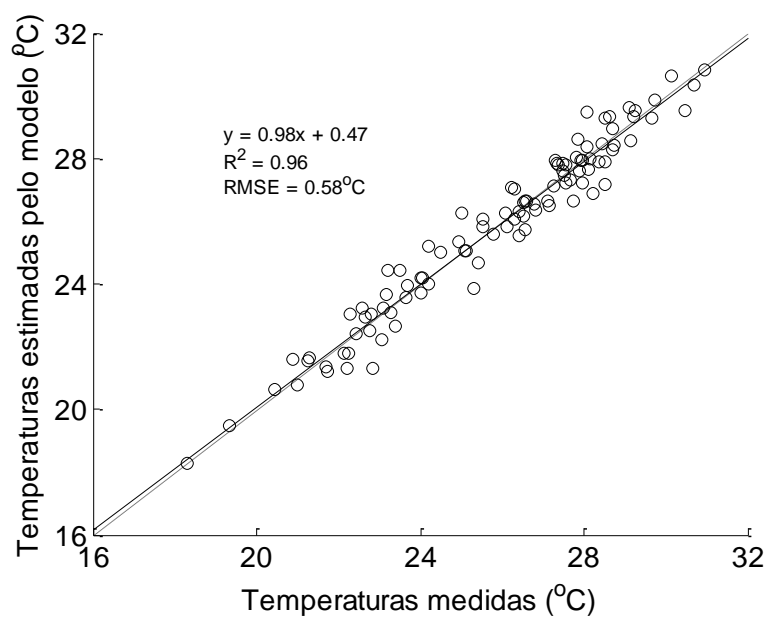

No período de verificação (07/01/2010 a 11/04/2010), o modelo se comportou tão bem quanto no período de calibração para ambas as variáveis (temperatura da coluna d'água e temperatura da superfície da água). Para a temperatura da coluna d'água, o coeficiente de correlação encontrado foi de 0,94 e a raiz do erro quadrático médio foi $0,40{ }^{\circ} \mathrm{C}$, com um erro médio de $-0,02{ }^{\circ} \mathrm{C}$. Para a temperatura da superfície, o coeficiente de determinação foi 0,97 , a raiz do erro quadrático médio foi 0,40 ${ }^{\circ} \mathrm{C}$ e o erro médio foi $0,02{ }^{\circ} \mathrm{C}$.

A habilidade do modelo DYRESM em estimar a evaporação do reservatório Logan também foi avaliada. Primeiramente, as evaporações totais diárias do modelo foram comparadas com a evaporação medida. As taxas de evaporação diárias estimadas pelo modelo também foram comparadas com as taxas de evaporação estimadas pelo método do balanço hídrico. A Figura 3 mostra a relação entre as medidas de evaporação por Eddy Covariance (EC) e as taxas estimadas por DYRESM. coeficiente de determinação $R^{2}$ foi 0,85 , com a raiz do erro quadrático médio $=0,57$ $\mathrm{mm} \cdot \mathrm{dia}^{-1}$ e erro médio $=-0,20 \mathrm{~mm} \cdot \mathrm{dia}^{-1}$.

O balanço hídrico foi computado considerando-se as entradas diárias de água no reservatório (precipitação direta) e as saídas (evaporação e percolação). As perdas por percolação foram assumidas como sendo constantes no tempo e igual a 1,5 $\mathrm{mm} \cdot \mathrm{dia}^{-1}$, conforme sugestão de um trabalho conduzido no reservatório Logan (MCJANNET et al., 2013), e de outros trabalhos conduzidos em reservatórios semelhantes na Austrália (CRAIG, et al., 2005; CRAIG; SCHMIDT; SCOBIE, 2006). O 
REA - Revista de estudos ambientais (Online) v.20, n. 2, p.62-76, jul./dez. 2018

balanço hídrico foi computado apenas para os períodos em que não houve enchimento artificial do reservatório e nem irrigação, para evitar a influência dos erros das medições destas variáveis nos valores de evaporação estimados pelo balanço hídrico. A Figura 4 mostra uma concordância satisfatória entre as taxas de evaporação diárias estimadas pelo balanço hídrico e as estimativas do modelo DYRESM. O coeficiente de determinação $R^{2}$ foi de 0,72 com a raiz do erro quadrático médio $=0,38 \mathrm{~mm} \cdot \mathrm{dia}^{-1}$, e erro médio de $-0,05 \mathrm{~mm} \cdot \mathrm{dia}^{-1}$.

Figura 3 - Correlação entre dados de evaporação medidos (eixo x) e dados estimados pelo modelo DYRESM (eixo y) no reservatório Logan. A linha sólida representa a equação de regressão, e a linha pontilhada a reta $\mathbf{y}=\mathbf{x}$

Fonte: Autores (2018)

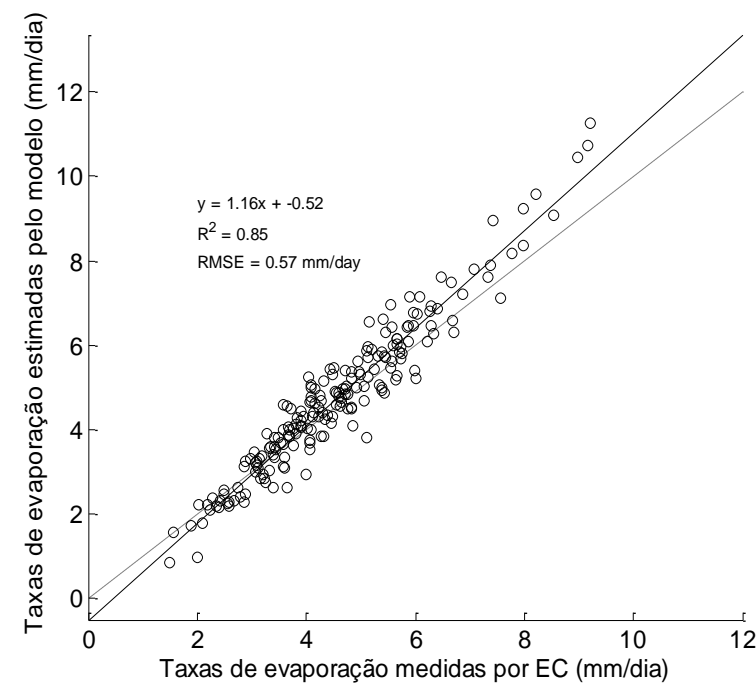

Figura 4 - Correlação entre dados de evaporação estimados por balanço hídrico (eixo x) e dados estimados pelo modelo DYRESM (eixo y) no reservatório Logan. A linha sólida representa a equação de regressão, e a linha pontilhada, a reta $y=x$

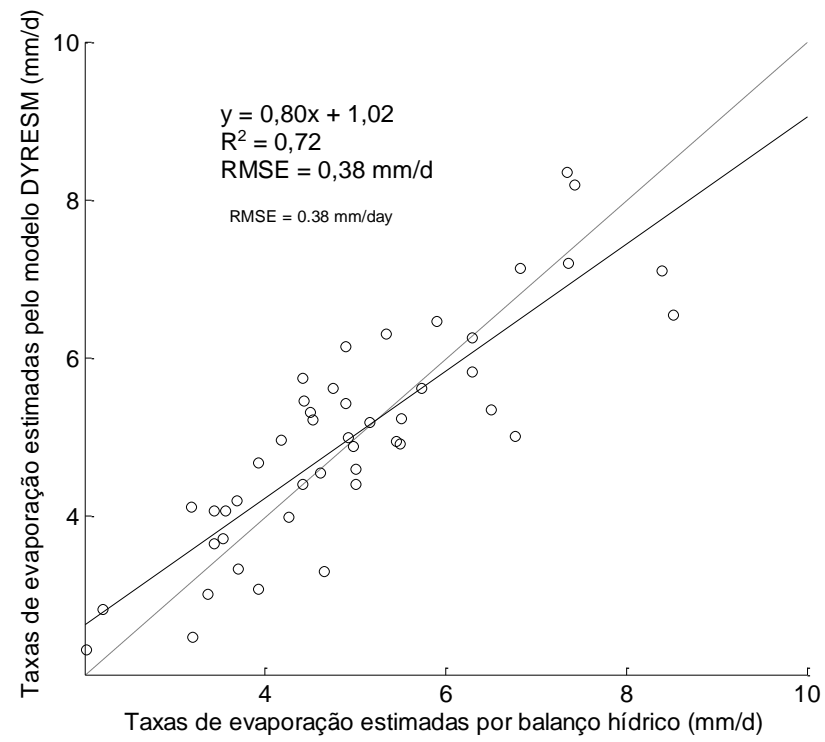

Fonte: Autores (2018) 


\section{REA - Revista de estudos ambientais (Online) v.20, n. 2, p.62-76, jul./dez. 2018}

Com base nos resultados acima, o modelo DYRESM foi considerado adequado para a previsão das temperaturas e das taxas de evaporação diárias do reservatório Logan. O modelo foi então aplicado para estimar as taxas de evaporação em condições normais (sem 0 uso de mecanismos de redução de evaporação) e sob condições de uso de coberturas suspensas, destratificadores e de quebraventos. A diferença nas taxas de evaporação em condições normais e com o uso de mecanismos de redução de evaporação forneceu uma indicação da eficácia dos mecanismos para reduzir a evaporação.

\section{4 - Incorporação dos mecanismos de redução de evaporação no modelo}

\subsection{1 - Coberturas suspensas}

Para representar o uso de coberturas suspensas no reservatório Logan, os seguintes parâmetros de entrada do modelo DYRESM foram alterados para refletir os efeitos do uso desta tecnologia na evaporação do reservatório: radiação solar incidente, cobertura de nuvens e velocidade do vento na superfície do lago. De acordo com Alvarez et al. (2006), o balanço de energia de radiação na superfície da água é influenciado pela transmissividade e pelas propriedades de absorção de calor do material usado como cobertura. De acordo com estes autores, uma cobertura porosa de camada simples de polietileno pode reduzir a radiação solar em até $80 \%$. Este fator foi aplicado neste estudo para alterar a radiação solar incidente. Além disso, o fator de sombreamento por nuvens no modelo DYRESM foi intensificado para representar o sombreamento proporcionado pela cobertura. Este fator é utilizado internamente pelo modelo para determinar a radiação de onda longa incidente no reservatório. A velocidade do vento na altura de 10 metros foi reduzida de acordo com a relação proposta por Yao et al. (2010) derivada de dados medidos por Alvarez et al. (2006) com a presença de cobertura suspensas sobre reservatórios:

$$
\mathrm{U}_{\text {cover er }}=0,086 \frac{\ln \left(2 / z_{\mathrm{p}}\right)}{\operatorname{In}\left(0,15 / z_{\mathrm{p}}\right)} \mathrm{U}
$$

onde $U_{\text {cover }}$ é a velocidade do vento equivalente a 10 metros de altura na presença de coberturas suspensas; $U$ é a velocidade do vento a 10 metros de altura em condições normais (sem cobertura); e $z_{0}$ é a rugosidade da superfície da água, assumida como sendo 0,004 m (HELFER; ZHANG; LEMCKERT, 2009).

\subsection{2 - Destratificadores}

Um algoritmo para modelar a temperatura da agua sob o uso de sistemas de destratificação artificial por injeção de ar foi incorporada no modelo DYRESM por Patterson e Imberger (1989). Esta sub-rotina é baseada no modelo de 'pluma simples' proposto por Mcdougall (1978), e utiliza a mesma discretização de camadas utilizada no modelo principal. Em cada intervalo de tempo, a pluma (mistura de ar e água) arrasta consigo uma quantidade de água de cada camada por onde ela passa. A pluma inicia-se na parte inferior da coluna de água, onde o ar é injetado, e arrasta consigo a água fria e densa do fundo. A pluma sobe na coluna de água até o ponto em que sua densidade equivale à densidade da coluna de água. A água densa e fria do fundo (D7) é arrastada para a superfície pelas bolhas de ar. Esta água é ejetada da pluma na superfície, proporcionando a mistura vertical. Este processo é esquematizado na Figura 5. A formulação usada no modelo DYRESM é descrita no manual científico do modelo (IMERITO, 2009a; b).

As variáveis de entrada na sub-rotina de destratificação artificial no DYRESM incluem profundidade e número de difusores em operação no lago, número de portas em cada difusor, e o fluxo de ar injetado por difusor em cada intervalo de tempo do modelo. Para o reservatório Logan, a taxa de injeção de ar total considerada foi de $0,01 \mathrm{~L}$ $\mathrm{s}^{-1}$ através de um difusor com 100 portas. $\mathrm{O}$ destratificador foi considerado em operação apenas nos meses de dezembro e janeiro, quando a demanda evaporativa do local é maior, e o reservatório possui reserva de água fria no fundo devido à estratificação térmica. As variáveis meteorológicas usadas foram as mesmas que as usadas nas condições naturais. 


\section{REA - Revista de estudos ambientais (Online) v.20, n. 2, p.62-76, jul./dez. 2018}

Figura 5 - Representação da mistura vertical proporcionada por destratificadores. a) Estratificação térmica natural de um reservatório, com densidade aumentando com a profundidade. b) reservatório em processo de destratificação artificial com a injeção de ar no fundo do lago

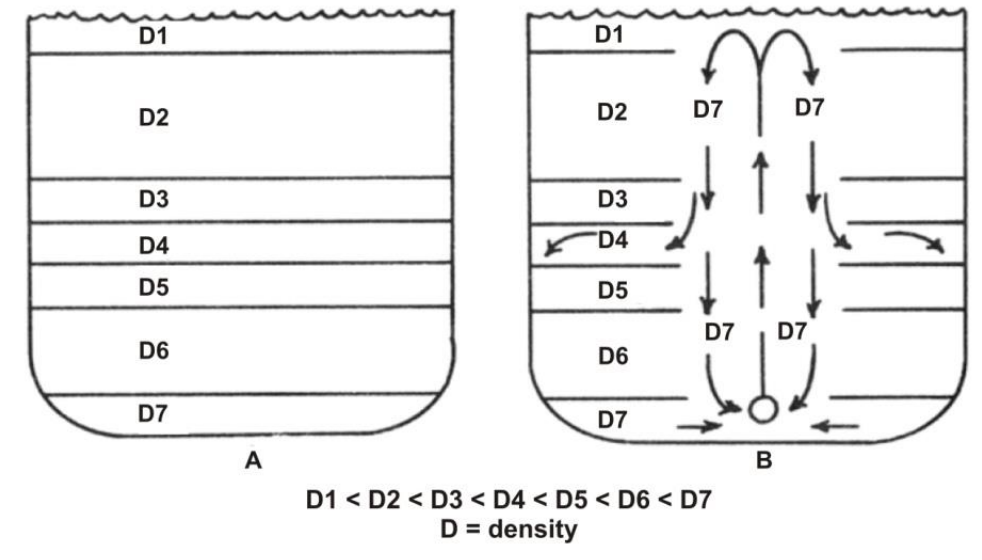

b)

Fonte: Adaptado de Fast (1968)

\subsubsection{Quebra-ventos}

De acordo com o diagrama mostrado na Figura 6, a redução do vento é máxima nos primeiros metros à jusante da barreira. $\mathrm{O}$ efeito do quebra-vento na velocidade do vento termina a uma distância de aproximadamente 30 vezes a altura do quebra-vento.

Neste estudo, a localização do reservatório Logan com relação ao quebravento foi considerada à jusante do quebravento, e as reduções na velocidade do vento na sua superfície foram consideradas conforme indicado na Figura 6. Considerouse um quebra-vento de 40 metros de altura e de porosidade média. Além disso, assumiuse que: i) as reduções na velocidade do vento mostradas na Figura 6 ocorreram uniformemente na direção vertical; ii) os quebra-ventos estavam posicionados perpendicularmente à direção predominante do vento; e iii) os quebra ventos estavam posicionados nas imediações da barragem, fazendo com que as reduções na velocidade do vento apresentadas na Figura 6 tivessem efeito apenas sobre a água, e não sobre a terra, no nível máximo do reservatório.

Figura 6 - Efeito de quebra-ventos de 20, 30 e 40 metros de altura e de porosidade média na velocidade do vento à jusante da barreira

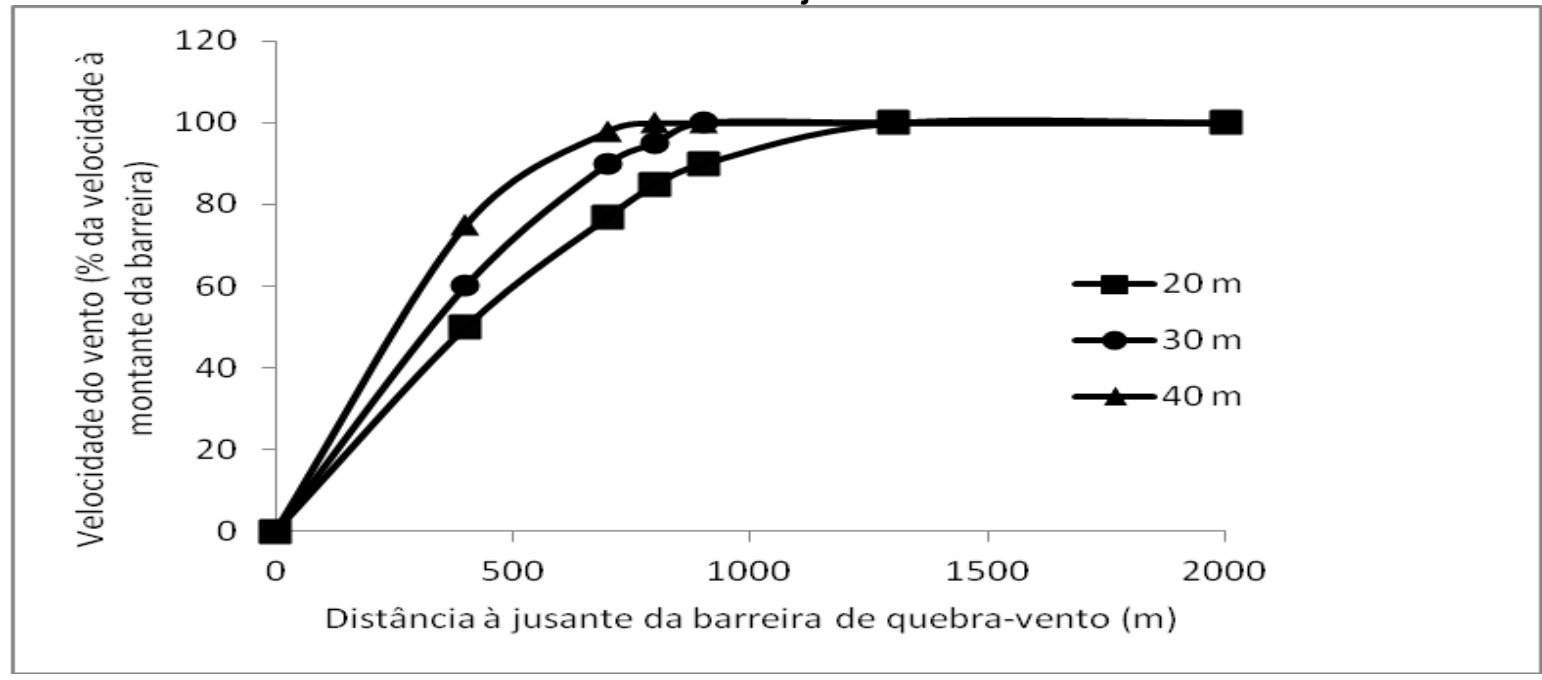

Fonte: Adaptado de Sudmeyer, Bicknell e Coles (2007) e Brandle, Hodges e Zhou (2004) 


\section{REA - Revista de estudos ambientais (Online) v.20, n. 2, p.62-76, jul./dez. 2018}

\section{Resultados}

O período de simulação neste estudo foi de 195 dias, começando na primavera, no dia 29 de setembro de 2009, e terminando no outono, no dia 11 de abril de 2010. A evaporação base obtida em condições normais (i.e. na ausência de mecanismos de redução de evaporação) foi de $906 \mathrm{~mm}$ para o período de 195 dias. As taxas variaram de $0,2 \mathrm{~mm} \cdot \mathrm{d}^{-1}$ a $11,1 \mathrm{~mm} \cdot \mathrm{d}^{-1}$. A série temporal das taxas de evaporação no período de estudo é apresentada na Figura 7.

Figura 7 - Taxas de evaporação diárias no reservatório Logan no período de estudo (29 de setembro de 2009 a 11 de abril de 2010)

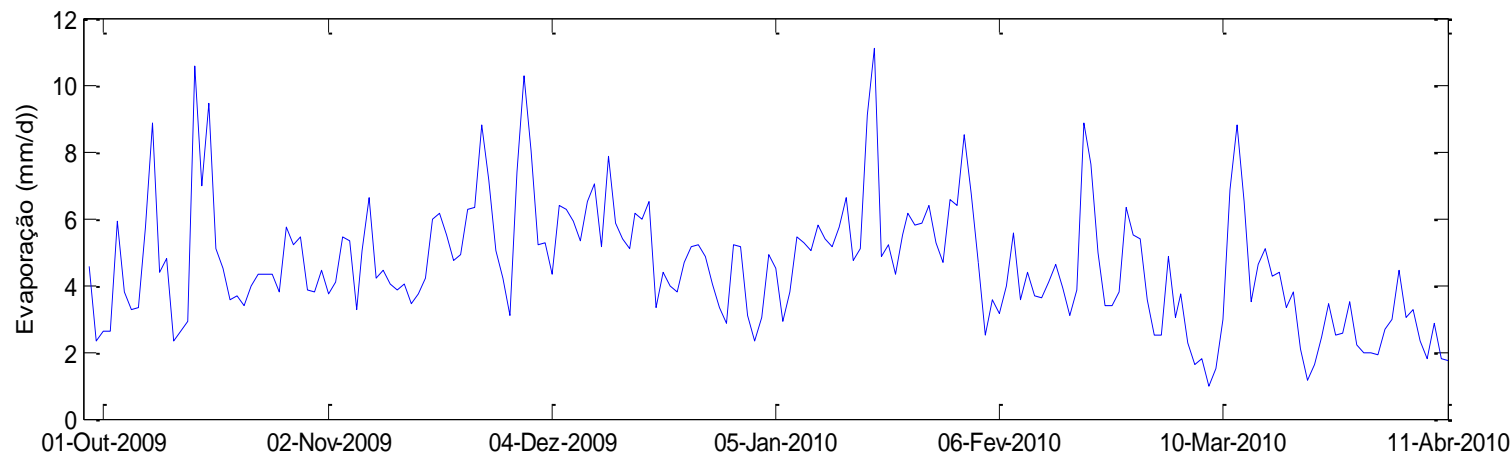

Fonte: Autores (2018)

As temperaturas da superfície do reservatório variaram de $20,6{ }^{\circ} \mathrm{C}$ a $31,1{ }^{\circ} \mathrm{C}$ (média de $26,6^{\circ} \mathrm{C}$ ) ao longo dos 195 dias de estudo. As temperaturas mais altas $\left(>30^{\circ} \mathrm{C}\right)$ ocorreram na segunda semana de dezembro de 2009 e na última semana de janeiro de 2010, em virtude das elevadas temperaturas do $\operatorname{ar}\left(>28^{\circ} \mathrm{C}\right)$ e taxas de radiação solar (> $310 \mathrm{~W} \mathrm{~m}^{-2}$ ). A temperatura média da água do fundo do reservatório foi de $25,2^{\circ} \mathrm{C}$. Por se tratar de um lago raso $(6,5$ metros de profundidade máxima), a ocorrência de estratificação térmica foi bastante rara, tendo sido observada apenas no início de novembro de 2009, segunda semana de dezembro de 2009 e no início de março de 2010, imediatamente após períodos de ventos fracos $\left(<1,5 \mathrm{~m} \mathrm{~s}^{-1}\right)$ e temperaturas altas $\left(>26^{\circ} \mathrm{C}\right)$. Estas condições atmosféricas levaram ao desenvolvimento de um gradiente térmico de cerca de $4,0{ }^{\circ} \mathrm{C} \mathrm{m} \mathrm{m}^{-1}$ nestas semanas. Apesar destes períodos de estratificação, a diferença entre as temperaturas da água de fundo e de superfície permaneceu baixa por todo 0 período de estudo $\left(<4,0^{\circ} \mathrm{C}\right)$.

A Figura 8 apresenta as evaporações acumuladas mensais para 0 cenário base e para os cenários com os mecanismos de redução.

Figura 8 - Evaporação acumulada mensal no reservatório Logan no cenário base e nos cenários com o uso de mecanismos de redução de evaporação

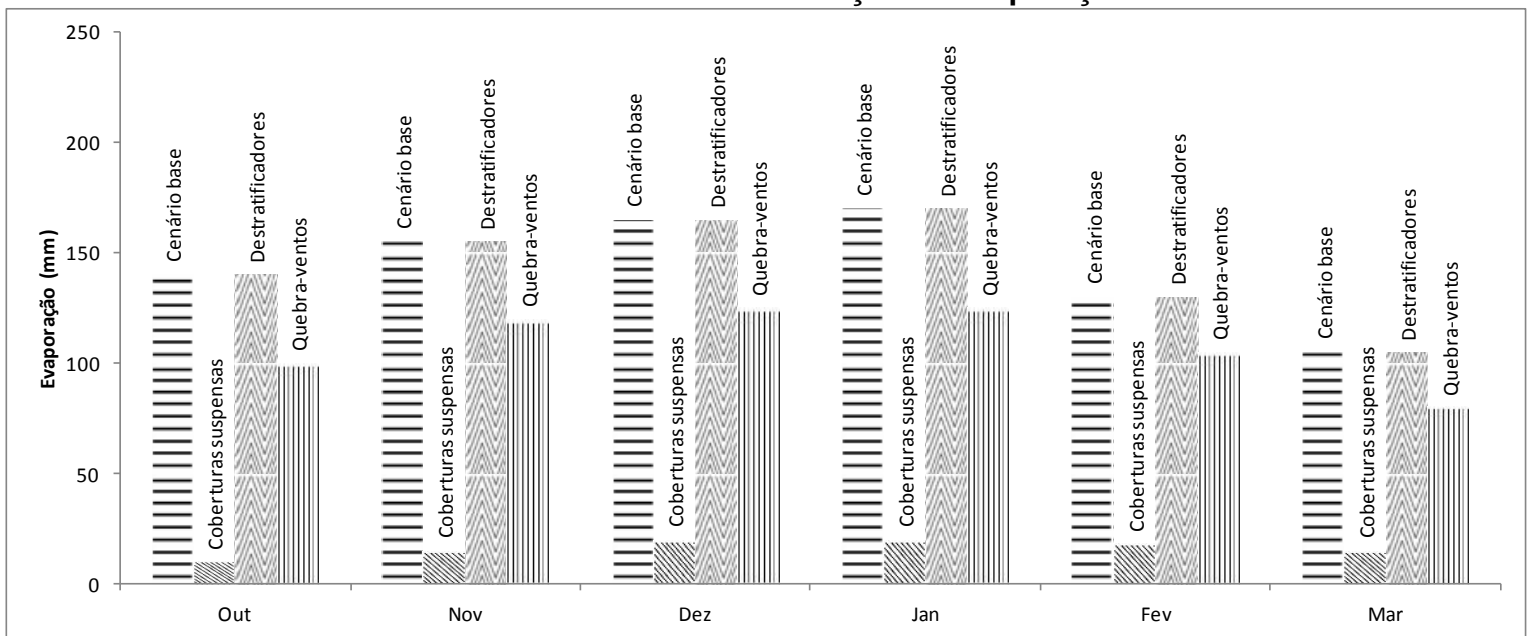

Fonte: Autores (2018) 


\section{REA - Revista de estudos ambientais (Online) v.20, n. 2, p.62-76, jul./dez. 2018}

Observa-se que a técnica mais eficaz na redução da evaporação foi o uso de coberturas suspensas, que reduziu a evaporação total do período em $88 \%$ com relação à evaporação base. Este resultado é consistente com os resultados encontrados por Alvarez et al. (2006) e Yao et al. (2010). Alvarez et al. (2006) mediram a evaporação de um tanque de evaporação sob o uso de coberturas suspensas e compararam estas medições com a evaporação medida de um tanque descoberto. A redução encontrada foi de $80 \%$. Yao et al. (2010) investigaram a eficácia de coberturas suspensas em um grande reservatório de abastecimento de água na Austrália usando uma versão modificada do modelo de Penman-Monteith, e encontraram uma redução de $91 \%$ com o uso da técnica. Estes resultados indicam não apenas que o uso de coberturas suspensas é um método bastante eficaz para redução de evaporação, mas também que a forma como o modelo DYRESM foi tratado para representar as coberturas suspensas é adequada, podendo a metodologia ser transferida para estudos semelhantes em outros reservatórios onde se queira estimar as reduções nas taxas de evaporação sob o uso de coberturas suspensas.

O excelente desempenho da técnica de coberturas suspensas em reduzir taxas de evaporação de reservatórios pode ser explicado pelo efeito combinado de dois fatores. O primeiro fator é que as coberturas suspensas reduzem a velocidade do vento sobre a água. $O$ vento tem um papel fundamental na evaporação, pois é responsável por remover o ar úmido que se acumula na superfície, mantendo o déficit de umidade na superfície constante, e aumentando a evaporação. Os resultados mostraram que a velocidade média do vento sobre a superfície do reservatório Logan caiu de 2,6 $\mathrm{m} \mathrm{s}^{-1}$ no cenário base, para 0,3 $\mathrm{m} \mathrm{s}-1$ no cenário com coberturas suspensas. O segundo fator, e o mais importante, é que as coberturas suspensas diminuem a quantidade de radiação que chega na superfície do reservatório, afetando assim a temperatura da água. A temperatura superficial é o parâmetro principal na determinação da quantidade de umidade (vapor de água) que o ar imediatamente acima da superfície pode conter. Temperaturas baixas estão relacionadas com baixo poder de contenção de umidade e, portanto, com reduzidas taxas de evaporação. A radiação solar direta foi reduzida em $70 \%$, e a temperatura média da superfície caiu de $26,6{ }^{\circ} \mathrm{C}$ no cenário de referência para $22,5{ }^{\circ} \mathrm{C}$ no cenário com coberturas. As temperaturas mais altas da superfície foram reduzidas com maior eficiência do que as temperaturas mais baixas. Por exemplo, a temperatura mais alta no período de estudo $\left(31,1^{\circ} \mathrm{C}\right)$ caiu para a $28^{\circ} \mathrm{C}(-10 \%)$ no cenário com coberturas suspensas. A temperatura mais baixa do período, de $20,6^{\circ} \mathrm{C}$, caiu para $19,5^{\circ} \mathrm{C}(-5 \%)$. Esse é outro fator importante na redução da evaporação, visto que temperaturas mais altas estão associadas com taxas de evaporação mais altas.

Os sistemas de destratificação foram os menos eficientes, com uma redução de $1,0 \%$ no total da evaporação. A temperatura média da superfície do reservatório não se alterou significativamente com 0 uso da aeração e, portanto, estes sistemas não influenciaram as taxas de evaporação. Isso ficou demonstrado através dos perfis térmicos observados em um dia com alto gradiente sem o uso de destratificadores. 0 efeito inicialmente esperado, como sugerido na literatura (HELFER; LEMCKERT, C.; ZHANG, 2011b; HELFER; LEMCKERT; ZHANG, 2011a; 2012b), era o de redução da temperatura da superfície devido a água fria do fundo do reservatório, carregada pela massa de ar injetada, misturar-se com a água quente da superfície. No entanto, o observado foi a temperatura do fundo do reservatório aumentando e as temperaturas da superfície mantendo-se inalteradas em condições de destratificação artificial. Este fenômeno pode ser explicado pela desestruturação da termoclina causada pela massa de ar injetada, o que favoreceu a mistura e o aprofundamento da camada superficial, continuamente aquecida pelas condições de superfície. Os resultados sugerem que o corpo de água ganhou calor sob condições de destratificação artificial.

$\mathrm{O}$ uso de quebra-ventos reduziu a evaporação do reservatório Logan em 27\%. A evaporação total no período de simulação com o uso destes sistemas foi de $661 \mathrm{~mm}$, em comparação com 906 mm no cenário de referência. A velocidade média diária do vento foi reduzida de $2,6 \mathrm{~m} \mathrm{~s}^{-1}$ para $1,5 \mathrm{~m} \mathrm{~s}^{-1}$. É importante notar, no entanto, que esta redução é esperada para um cenário onde o vento atua perpendicularmente à orientação da barreira, e a barreira é implementada na borda imediata da barragem. Portanto, na prática, a redução seria provavelmente inferior, pois a direção do vento altera-se com frequência e nem sempre é possível 


\section{REA - Revista de estudos ambientais (Online) v.20, n. 2, p.62-76, jul./dez. 2018}

estabelecer o quebra-vento na proximidade da barragem, devido ao risco de vazamento e até mesmo de rompimento da barragem por causa de presença de raízes.

A orientação do quebra-vento em relação ao reservatório também é um fator importante. Se o reservatório for largo e estreito, e a barreira estiver posicionada ao longo do lado de maior dimensão, o efeito esperado será maior (maiores reduções de evaporação) do que se a barreira estiver posicionada ao longo do lado de menor dimensão do reservatório. Isso é porque, conforme apresentado na Figura 6 , as reduções na velocidade do vento ocorrem sobre uma área à jusante do quebra-vento cuja distância é limitada (dependente da altura e porosidade do quebra-vento). Para maximizar o efeito desta técnica em reduzir taxas de evaporação, na impossibilidade de instalar-se quebra-ventos ao redor de todo o perímetro do reservatório, os quebra-ventos deveriam ser posicionados ao longo do lado de maior dimensão. Um estudo semelhante, por exemplo, foi conduzido em um reservatório cujo comprimento era de cerca de 10 vezes a largura (HELFER; ZHANG; LEMCKERT, 2009). Simulações mostraram que, com os quebra-ventos posicionados ao longo da dimensão menor (largura) a redução na evaporação seria de apenas $4,0 \%$. Por outro lado, se os quebra-ventos fossem posicionados ao longo da dimensão maior (comprimento), as reduções seriam de $20 \%$.

Outro fator a considerar é o tamanho do reservatório. Visto que as reduções no vento são observadas apenas sobre uma distância limitada à jusante da barreira (a qual é determinada pela altura e densidade da barreira) o ideal seria que o reservatório não ultrapassasse este limite, pois qualquer área fora desta área de influência do quebravento não sofrerá alteração na evaporação. É por isso que quebra-ventos têm sido recomendados apenas para pequenos reservatórios (HIPSEY, 2002; HIPSEY; SIVAPALAN, M.; CLEMENT, 2004).

\section{Conclusões}

Os resultados mostraram que a aplicação de coberturas suspensas é o mecanismo mais eficaz para reduzir a evaporação de pequenos reservatórios, com uma redução esperada de aproximadamente $88 \%$. Esta eficácia pode variar dependendo do material utilizado como cobertura e da altura de instalação. $O$ uso de quebra-ventos proporcionou reduções potenciais de $27 \%$. Espera-se que esta redução seja menor na prática, onde nem sempre as condições estabelecidas para este estudo podem ser replicadas, como por exemplo, o posicionamento estratégico do quebra-vento em relação ao vento predominante. A utilização de sistemas de destratificação artificial pode ser descartada como um método para redução de evaporação de reservatórios de pequeno porte (rasos). Estes sistemas talvez tenham maior efeito em reservatórios mais profundos onde a quantidade de água fria armazenada no fundo é maior. Um estudo comparativo de custo-benefício de coberturas suspensas e quebra-ventos é recomendado, uma vez que os últimos parecem incorrer em custos de instalação e manutenção mais baixos, além de menores impactos ambientais.

No geral, os resultados deste trabalho são promissores, pois indicam a existência de mecanismos eficazes e viáveis para reduzir a evaporação de reservatórios de pequeno porte. A eficácia de cada mecanismo vai depender das condições atmosféricas de cada reservatório, o que indica que estudos como este devem ser conduzidos antes de definir uma técnica para cada local em questão. Além disso, a escolha dependerá também da relação custo-benefício e dos impactos ambientais associados com cada técnica, confirmando que análises locais devem ser conduzidas para uma tomada de decisão adequada.

\section{A Comparative Study of Three Mechanisms of Reservoir Evaporation Control}

\footnotetext{
Abstract: The use of technologies to reduce reservoir evaporation can help arid and semi-arid regions to overcome water scarcity. The most widespread mechanisms, however, such as the use of monomolecular films and floating structures, may impose stress on aquatic environments and water quality. This study aimed to investigate the effectiveness of three evaporation reduction techniques that have high environmental performance: suspended covers, destratification systems and windbreaks. The analysis was conducted using the model DYRESM that was calibrated and validated for a reservoir located in the South-East Queensland
} 


\section{REA - Revista de estudos ambientais (Online) v.20, n. 2, p.62-76, jul./dez. 2018}

region in Australia. The boundary conditions employed in the model were changed to reflect the effects of the evaporation reduction techniques on the thermal behavior of the reservoir under study. The most effective method was the use of suspended covers, which reduced evaporation by $88 \%$. Windbreaks reduced evaporation by $27 \%$, and destratification systems had no significant effect on the evaporation rates. For each technology, a comprehensive analysis of their effect on water temperature and evaporation was performed in order to make an inference of the expected effects of these techniques when used in reservoirs under other climatic conditions.

Keywords: Evaporation; DYRESM; Suspended covers; Destratification systems; Windbreaks.

\section{Referências}

ABC RURAL. Millions of little plastic balls could stop evaporation from water storages, 2015.

ALVAREZ, V. M.; BAILLE, A.; MARTÍNEZ, J. M. M.; GONZALES-REAL, M. M. Effect of black polyethylene shade covers on the evaporation rate of agricultural reservoirs. Spanish Journal of Agricultural Research, p. 280-288, 2006.

BARNES, G. T. Optimum conditions for evaporation control by monolayers. Journal of Hydrology, v. 145, p. 165-173, 1993.

BRANDLE, J. R.; HODGES, L.; ZHOU, X. H. Windbreaks in North American agricultural systems. Agroforestry Systems, v. 61, p. 65-78, 2004.

CRAIG, I. Evaporation control using covers. 15th Queensland Water Symposium (IEAust Water Panel), 2005.

CRAIG, I.; GREEN, A.; SCOBIE, M.; SCHMIDT, E. Controlling Evaporation Loss from Water Storages: National Centre for Engineering in Agriculture, Publication 1000580/1, Toowoomba 2005.

CRAIG, I.; SCHMIDT, E.; SCOBIE, M. Evaporation control using covers - some realistic solutions for the irrigation industry. Irrigation Australia 2006: Irrigation Association of Australia National Conference and Exhibition. Brisbane, Australia 2006.

CSIRO; BOM. Climate Change in Australia. Technical report of CSIRO, Bureau of Meteorology and the Australian Greenhouse Office in partnership with the Australian Climate Change Science Program, Canberra, 148 p., 2007. Disponível em: www.climatechangeinaustralia.gov.au/technical_re port.php. Acesso em: 10 mar. 2018.

MELO BRANCO, A.; SUASSUNA, J.; VAINSENCHER, S. A. Improving Access to Water Resources through Rainwater Harvesting as A Mitigation Measure: The Case of the Brazilian Semi-Arid Region. Mitigation and Adaptation Strategies for Global Change, v. 10, n. 3, p. 393409, 2005.
DEPARTMENT OF NATURAL RESOURCES AND MINES. Current knowledge and developing technology for controlling evaporation from on-farm storage: Report for the National Program for Irrigation Research and Development, Brisbane 2002.

DEPARTMENT OF PRIMARY INDUSTRIES. Water savings in the Darling River including the Menindee Lakes, 2015.

FAST, A. W. Artificial Destratification of EI Capitan Reservoir by Aeration. Part I: Effects on Chemical and Physical Parameters: Bulletin 141, Department of Fish and Game, California, 1968.

FINN, N.; BARNES, S. The benefits of shadecloth covers for potable water storages. CSIRO Textile and Fibre Technology. 2007.

FISHER, H. B.; LIST, E. J.; KOH, R. Y. C.; IMBERGER, J.; BROOKS, N. H. Mixing in Inland and Coastal Waters. New York: Academic Press, 1979.

FONTES, A. S.; OLIVEIRA, J. I. R. D.; MEDEIROS, Y. D. P. A evaporação em açudes no semi-árido nordestino do Brasil e a gestão das águas. In: XV Simpósio Brasileiro de Recursos Hídricos. Curitiba, Brasil: ABRH 2003.

GALLEGO-ELVIRA， B.; MARTIINEZ-ALVAREZ, V.; PITTAWAY, P.; BRINK, G.; MARTÍN-GORRIZ, B. Impact of Micrometeorological Conditions on the Efficiency of Artificial Monolayers in Reducing Evaporation. Water Resources Management, v. 27, n. 7, p. 2251-2266, 2013.

HELFER, F.; LEMCKERT, C.; ZHANG, $H$. Assessing the effectiveness of air-bubble plume aeration in reducing evaporation from farm dams in Australia using modelling. In: BREBBIA, C. A. e POPOV, V. (Ed.). Water Resources Management VI: WIT Press, United Kingdom, 2011a. p. 485496.

HELFER, F.; LEMCKERT, C.; ZHANG, $H$. Modelling of lake mixing induced by air-bubble plumes and the effects on evaporation. Journal of Hydrology, v. 406, p. 182-198, 2011 b.

HELFER, F.; LEMCKERT, C.; ZHANG, H. Impacts of climate change on temperature and evaporation 


\section{REA - Revista de estudos ambientais (Online) v.20, n. 2, p.62-76, jul./dez. 2018}

from a large reservoir in Australia. Journal of Hydrology, v. 475, n. 0, p. 365-378, 2012a.

HELFER, F.; LEMCKERT, C.; ZHANG, $H$. Influence of bubble plumes on evaporation from non-stratified waters. Journal of Hydrology, v. 438, p. 84-96, 2012b.

HELFER, F.; ZHANG, H.; LEMCKERT, C. Evaporation reduction by windbreaks: overview, modelling and efficiency. Technical Report no. 16 for the Urban Water Security Research Alliance, Brisbane. 2009.

HIPSEY, M. Using windbreaks to reduce evaporation from farm dams, Department of Agriculture WA, Farmnote, 2002.

HIPSEY, M. R.; SIVAPALAN, M.; CLEMENT, T. P. A numerical and field investigation of surface heat fluxes from small wind-sheltered waterbodies in semi-arid Western Australia. Environmental Fluid Mechanics, v. 4, p. 79-106, 2004.

IMBERGER, J.; PATTERSON, J. C. A dynamic reservoir simulation model - DYRESM. In: FISCHER, H. B. (Ed.). Transport Models for Inland and Coastal Waters. New York: Academic Press, 1981. p. 310-361.

IMERITO, A. Dynamic Reservoir Simulation Model (DYRESM) v4.0 - Science Manual. Centre for Water Research, The University of Western Australia. 2009a.

IMERITO, A. Dynamic Reservoir Simulation Model (DYRESM) v4.0 - User Guide. Centre for Water Research, University of Western Australia. 2009b.

JOHNSON, F.; SHARMA, A. A Comparison of Australian Open Water Body Evaporation Trends for Current and Future Climates Estimated from Class A Evaporation Pans and General Circulation Models. Journal of Hydrometeorology, v. 11, n. 1, p. 105-121, 2010.

KIRK, J. T. O. The Vertical Attenuation of Irradiance as a Function of the Optical Properties of the Water. Limnology and Oceanography, v. 48 , n. 1, p. 9-17, 2003.

KLING, G. W. Comparative transparency, depth of mixing, and stability of stratification in lakes of Cameroon, West Africa. Limnology and Oceanography, v. 33, n. 1, p. 27-40, 1988.

LEÃO, R. A. O.; SOARES, A. A.; TEIXEIRA, A. D. S.; DA SILVA, D. D. Estimation of evaporation in the banabuiú dam, in the state of ceará, brazil, by different combined methods, derived from the penman equation. Engenharia Agricola, v. 33, n. 1, p. 129-144, 2013.

MAESTRE-VALERO, J. F.; MARTÍNEZGRANADOS, D.; MARTÍNEZ-ALVAREZ, V.;
CALATRAVA, J. Socio-Economic Impact of Evaporation Losses from Reservoirs Under Past, Current and Future Water Availability Scenarios in the Semi-Arid Segura Basin. Water Resources Management, v. 27, n. 5, p. 1411-1426, 2013.

MARTÍNEZ ALVAREZ, V.; GONZÁLEZ-REAL, M. M.; BAILLE, A.; MAESTRE VALERO, J. F.; GALLEGO ELVIRA, B. Regional assessment of evaporation from agricultural irrigation reservoirs in a semiarid climate. Agricultural Water Management, v. 95, n. 9, p. 1056-1066, 2008.

MCDOUGALL, T. J. Bubble plumes in stratified environments. Journal of Fluid Mechanics, v. 85, p. 655-672, 1978.

MCGLOIN, R.; MCGOWAN, H.; MCJANNET, D.; BURN, S. Modelling sub-daily latent heat fluxes from a small reservoir. Journal of Hydrology, $v$. 519, Part B, p. 2301-2311, 2014.

MCGLOIN, R.; MCGOWANA, H.; MCJANNET, D. Effects of diurnal, intra-seasonal and seasonal climate variability on the energy balance of a small subtropical reservoir. International Journal of Climatology, v. 35, p. 2308-2325, 2015.

MCJANNET, D.; COOK, F.; BURN, S. Evaporation Reduction by Manipulation of Surface Area to Volume Ratios: Overview, Analysis and Effectiveness. Technical Report 8 for the Urban Water Security Research Alliance, Brisbane. 2008.

MCJANNET, D.; COOK, F.; MCGLOIN, R.; MCGOWAN, H.; BURN, S.; SHERMAN, B. Longterm energy flux measurements over an irrigation water storage using scintillometry. Agricultural and Forest Meteorology, v. 168, p. 93-107, 2013.

OLIVER, R. L.; HART, B. T.; OLLEY, J.; GRACE, M.; REES, C.; CAITCHEON, G. The Darling River: Algal Growth and the Cycling and Sources of Nutrients. Murray Darling Basin Commission, Project M386, Final Report. 2000.

PATTERSON, J. C.; IMBERGER, J. Simulation of bubble plume destratification systems in reservoirs. Aquatic Sciences, v. 51, n. 1, p. 3-18, 1989.

PAZ, V. P. D. S.; TEODORO, R. E. F.; MENDONÇA, F. C. Recursos hídricos, agricultura irrigada e meio ambiente. Revista Brasileira de Engenharia Agrícola e Ambiental, v. 4, n. 3, p. 465-473, 2000

PEREIRA, S. B.; PRUSKI, F. F.; SILVA, D. D. D.; RAMOS, M. M. Evaporação líquida no lago de Sobradinho e impactos no escoamento devido à construção do reservatório. Revista Brasileira de Engenharia Agrícola e Ambiental, v. 13, n. 3, p. 346-352, 2009 


\section{REA - Revista de estudos ambientais (Online) v.20, n. 2, p.62-76, jul./dez. 2018}

PERROUD, M.; GOYETTE, S.; MARTYNOV, A.; BENISTON, M.; ANNEVILLE, O. Simulation of multiannual thermal profiles in deep Lake Geneva: a comparison of one-dimensional lake models. Limnology and Oceanography, v. 54, p. 1574 1594, 2009

PRIME, E.; LEUNG, A.; TRAN, D.; GILL, H.; SOLOMON, D.; QIAO, G.; DAGLEY, I. New technology to reduce water evaporation from large water storages. Canberra, p.48. 2012.

REBOUÇAS, A. C. Água na região Nordeste: desperdício e escassez. Estudos Avançados, v. 11, n. 29, p. 127-154, 1997.

ROSEGRANT, M. W.; RINGLER, C.; ZHU, T. Water for Agriculture: Maintaining Food Security under Growing Scarcity. Annual Review of Environment and Resources, v. 34, n. 1, p. 205222, 2009.

SADEGHI, S.-H.; PETERS, T. R.; AMINI, M. Z.; MALONE, S. L.; LOESCHER, H. W. Novel approach to evaluate the dynamic variation of wind drift and evaporation losses under moving irrigation systems. Biosystems Engineering, v. 135 , p. $44-53,2015$

SCHMIDT, E. Controlling Evaporation Loss from Water Storages using NetPro Shade cloth. Toowoomba: National Centre for Engineering in Agriculture, Publication 1000580/3, 2007.

SILANS, A. M. B. P. D. Redução da Evaporação de Açudes - $O$ Estado da Arte. Revista Brasileira de Recursos Hídricos, v. 8, n. 2, p. 101-109, 2003.

SUASSUNA, J. A Pequena e Média Açudagem no Semi-árido Nordestino: uso da água para produção de alimentos. Artigos - João Suassuna, Recife, Brasil, 2002.

SUDMEYER, R.; BICKNELL, D.; COLES, N. Tree windbreaks in the wheatbelt. Department of Agriculture and Food, Government of Western Australia, Bulletin 4723. 2007.

SUDMEYER, R. A.; SCOTT, P. R. Characterisation of a windbreak system on the south coast of Western Australia. 1. Microclimate and wind erosion. Australian Journal of Experimental Agriculture, v. 42, p. 703-715, 2002.

TANENTZAP, A. J.; HAMILTON, D. P.; YAN, N. D. Calibrating the Dynamic Reservoir Simulation
Model (DYRESM) and filling required data gaps for one-dimensional thermal profile predictions in a boreal lake. Limnology and Oceanography: Methods, v. 5, p. 484-494, 2007.

URBAN WATER SECURITY RESEARCH ALLIANCE. 5 Years of Urban Water Research in South East Queensland - 2007-2012. Brisbane, p.32. 2012. Disponível em: http://www.urbanwateralliance.org.au/. Acesso em: 08 mar. 2018.

WANG, H.; TAKLE, E. S. Model-Simulated Influences of Shelterbelt Shape on WindSheltering Efficiency. Journal of Applied Meteorology, v. 36, n. 6, p. 695-704, 1997.

WATTS, P. J. Scoping study: reduction of evaporation from farm dams. FINAL REPORT TO THE NATIONAL PROGRAM FOR SUSTAINABLE IRRIGATION. Toowoomba: Feedlot Services Australia Pty Ltd 2005.

WEINBERGER, S.; VETTER, M. Using the hydrodynamic model DYRESM based on results of a regional climate model to estimate water temperature changes at Lake Ammersee. Ecological Modelling, v. 244, p. 38-48, 2012.

WEINBERGER, S.; VETTER, M. Lake heat content and stability variation due to climate change: coupled regional climate model (REMO)lake model (DYRESM) analysis. Journal of Limnology, v. 73, n. 1, 2014-02-19 2014.

YAO, X.; ZHANG, H.; LEMCKERT, C.; BROOK, A.; SCHOUTEN, P. Evaporation Reduction by Suspended and Floating Covers: Overview, Modelling and Efficiency. Technical Report No. 28 for the Urban Water Security Research Alliance, Brisbane. 2010.

\section{Agradecimentos}

Os autores agradecem 0 apoio financeiro do CNPq (Brasil), do Instituto de Pesquisas Hidráulicas da Universidade Federal do Rio Grande do Sul (Brasil) e da School of Engineering da Griffith University (Austrália). Os autores também agradecem a Urban Water Research Security Alliance (Austrália) por providenciar os dados para este estudo, e o Centre for Water Research da University of Western Australia por fornecer o modelo DYRESM usado. 